To the Editors:

\title{
Study on Body Mass Index, waist circumference and waist to height ratio of nursing officers working at Teaching Hospital, Jaffna
}

\author{
S Sasrubi ${ }^{1}$, S Balakumar ${ }^{2}$, V Arasaratnam ${ }^{2}$ \\ Ceylon Medical Journal 2018; 63: 88-89 \\ DOI: http://doi.org/10.4038/cmj.v63i2.8674
}

Body Mass Index (BMI), waist circumference and waist-to-height ratio are simple tools to assess obesity, and obesity related, metabolic and cardio vascular disease risk status [1]. The aim of this study was to find the prevalence of overweight and obesity among the nursing officers working at Teaching Hospital, Jaffna. As the Teaching Hospital, Jaffna has 1500 nursing officer cadres and only 396 cadres are filled, it was expected that the working environment and pattern of duty may interfere with their regular eating habits, sleeping pattern and physical exercise and lead to alteration in BMI, waist circumference and waist-to-height ratio of the nursing officers. An institutional based cross-sectional descriptive study was carried out during February 2013. Ethics approval was obtained from the Ethical Review Committee of the Faculty of Medicine, University of Jaffna. Body weight was measured using an electronic weighing scale (Seca), height was measured using a stadiometer (Seca) and waist circumference was measured using a nonstretchable measuring tape following standard procedure $[2,3]$. BMI was classified as underweight, normal weight, overweight, obesity class I, obesity class II and obesity class III [4]. Participants with waist circumference $>90.0 \mathrm{~cm}$ in males or $80.0 \mathrm{~cm}$ in females were considered to have central obesity [3]. Waist-to-height ratio value above 0.5 is an indicator of 'early heath risks' associated with central obesity [3]. The Teaching Hospital, Jaffna has 130 (32.8\%) male and $266(67.2 \%)$ female nursing officers and out of them $118(34.5 \%)$ males and $224(65.5 \%)$ females responded. The mean body weight of males was $69.3( \pm 11.8) \mathrm{kg}$ and $58.6( \pm 10.8) \mathrm{kg}$ in females. The mean BMI of males was $24.1( \pm 3.5)$ and in females $24.1( \pm 4.3) \mathrm{kgm}-2$. Among the male nursing officers, $6(5.1 \%)$ were underweight, 65 $(55.1 \%)$ had normal weight, $40(33.9 \%)$ were overweight and $7(5.9 \%)$ were obesity class- I. Among the female nursing officers 17 (7.6\%) were underweight, $123(54.9 \%)$ were normal weight, $63(28.1 \%)$ were overweight, $18(8.0 \%)$ were obesity class -I and $3(1.3 \%)$ were obesity class -II. Prevalence of obesity was higher among the female nurses than the male nurses and obesity class- II was found only among female nurses. Previous study carried out in Sri Lanka reports that $18.2,52.4,22.8$, and $6.6 \%$ of the adult women were of underweight, normal weight, over weight and Obesity Class- I respectively [5]. Rates of overweight and obesity were more among female nurses compared to the general population [5]. Based on the BMI values 39.8 of males and and $37.4 \%$ of females were either overweight or obese. Based on waist circumference, $50.8 \%$ of males

Table 1. Ranges of BMI, waist circumfrence and waist-to-height ratio among male and female nurses working at Teaching Hospital, Jaffna

\begin{tabular}{lcccc}
\hline Gender & Body Weight $(\mathrm{kg})$ & BMI $\left(\mathrm{kg} / \mathrm{m}^{2}\right)$ & Waist circumference $(\mathrm{cm})$ & Waist-to-hip ratio \\
\hline Male & $45.0-100.0$ & $16.5-33.3$ & $68.0-111.0$ & $0.4-0.7$ \\
Female & $35.2-90.5$ & $15.2-37.9$ & $60.0-119.0$ & $0.4-0.8$ \\
Total & $35.2-100.0$ & $15.2-37.9$ & $60.0-119.0$ & $0.4-0.8$ \\
\hline
\end{tabular}

${ }^{1}$ Allied Health Sciences Unit, Faculty of Medicine, University of Jaffna, ${ }^{2}$ Department of Biochemistry, Faculty of Medicine, University of Jaffna, Sri Lanka.

Correspondence: SS, e-mail: <sasrubi@hotmail.co.uk>. Received 05 May 2018 and revised version accepted 09 May 2018. 
and $70.5 \%$ of females had central obesity and based on waist-to-height ratio $70.3 \%$ of males and $74.6 \%$ of females were at high risk. This study shows that even though the BMI values are considered in classifying overweight and obesity, waist circumference and waist-to-height ratio may be more useful to identify health risk. This study indicated that at Teaching Hospital, Jaffna overweight and obesity was more among female nurses than males and these rates were higher than the national values.

\section{References}

1. World Health Organization. Obesity and overweight, Fact Sheet Number 311, World Health Organization 2012.
2. Taking Height, Weight and BMI measurements; Tools for personal Training Success; www.ptdirect.com

3. WHO Expert Consultation Appropriate body-mass index for Asian populations and its implications for policy and intervention strategies. The Lancet 2004; 363(9403): 157-63.

4. The International Diabetes Federation consensus worldwide definition of the metabolic syndrome. International Diabetes Federation 2006.

5. Jeyatissa R, Gunawardana S, Gunathilaka M, Hossain SM, Ranbanda JM, Silva PC. Prevalence and associations of overweight among adult women in Sri Lanka: a national survey. 2012: Sri Lanka Journal of Diabetes Endocrinology and Metabolism 2(2): 61-8. 